\title{
Absorption of impinging water droplet in
}

\section{porous stones}

\author{
J. B. Lee ${ }^{1}$, A. I. $\operatorname{Radu}^{1,2}$, P. Vontobel ${ }^{3}$, D. Derome ${ }^{2}$, J. Carmeliet $^{1,2^{*}}$
}

${ }^{1}$ Chair of Building Physics, ETH Zürich, Stefano-Franscini-Platz 5, CH-8093 Zürich, Switzerland

${ }^{2}$ Laboratory for Multiscale Studies in Building Physics, Swiss Federal Laboratories for Materials

Science and Technology, Empa, Überlandstrasse 129, CH-8600 Dübendorf, Switzerland

${ }^{3}$ Laboratory for Neutron Scattering and Imaging, Paul Scherrer Institut (PSI), Villigen, Switzerland

\begin{abstract}
This paper presents an experimental investigation and numerical analysis of the absorption of water droplets impacting porous stones. The absorption process of an impinging droplet is here fully characterized from spreading to evaporation in terms of absorbed mass during droplet depletion and moisture content distribution in a time-resolved manner for three different natural stones. High-speed imaging and neutron radiography are used to quantify moisture absorption in porous stones of varying moisture properties from deposition until depletion. During impact and spreading, the droplet exhibits a dynamic non-wetting behavior. At maximum spreading, the droplet undergoes pinning, resulting into the contact radius remaining constant until droplet depletion. Absorption undergoes two phases: initially, absorption is hindered due a contact resistance attributed to entrapped air; afterwards, a more perfect capillary contact occurs and absorption goes on until depletion, concurrently with evaporation and further redistribution. A finite-element numerical model for isothermal unsaturated moisture transport in porous media captures the phases of mass absorption in good agreement with the experimental data. Droplet spreading and absorption are highly determined by the impact velocity of the droplet, while moisture content redistribution after depletion is much less dependent on impact conditions.
\end{abstract}

Keywords: droplet absorption; porous natural stone; neutron imaging; droplet impact; transport modeling

\section{Introduction}

The phenomenon of drop impact on porous media is ubiquitous in nature and is associated with mechanisms found in various industrial applications [Yarin 2006, Gambaryan-Roisman 2014].When a liquid droplet impacts on a permeable surface, it spreads on the surface and is absorbed into the 
porous material due to capillary action. The spreading behavior of the impinging droplet on the surface is known to depend on the properties of the liquid, i.e. density, viscosity and surface tension, impact conditions such as drop size and impact velocity, and the surface wettability and roughness [Yarin 2006]. Absorption is governed both by the properties of the liquid and of the porous medium, i.e. porosity, pore size, wettability [Hapgood et al. 2002]. Once the deposited droplet is completely depleted from the surface, the liquid further redistributes within the porous medium due to capillary forces and evaporation occurs at the surface [Reis et al. 2003].

Although spreading of drop impacting solid impervious surfaces has been the subject of numerous experimental, analytical and numerical studies over the last few decades as presented in two review papers [Yarin 2006, Marengo et al. 2011], the fate of droplets impacting porous media is still far from being understood. Such study needs to consider jointly the droplet dynamics and the porous medium characteristics. In a recent study, we have identified that the spreading of impacting droplets on porous stones is accompanied by hydrophobic dynamic contact angles and a pinning of the droplet at maximum spreading [Lee et al. 2015,2016].

However, the absorption of deposited droplets on porous media is much more rarely studied. Given the difficulty of knowing the status of the liquid within the porous media, absorption studies focused first on the depleting droplet, such as in Denesuk et al. [1993] where the contact radius variation during droplet depletion is modeled. Similarly, Clarke et al. [2002] determined the absorption of deposited droplet into microporous filter membranes by measuring the volume of deposited droplet remaining on the surface and proposed an analytical model using Lucas-Washburn equation, assuming only vertical liquid uptake and using a constant permeability. Hapgood et al. [2006] investigated the depletion time of droplets deposited on various powder bed substrates and compared the experimental results with the analytical model from Denesuk et al. [1993] by taking into account the measured pore size and wetting properties of the powder bed substrates. However, with different means, described next, of investigating the liquid distribution within the porous media, several experimental studies showed that droplet absorption does not follow Washburn's law for liquid uptake in granular media [Reis et al. 2003, D’Onofrio et al. 2010, Jung et al, 2012]. Using a porous medium, Ben Jazia et al. [2011] also showed deviations from the expected square root time-dependence as shown by Washburn's law with experiments of drop absorption into hydrophilic nanoporous media formed by polystyrene microbeads assemblies. For this work, they calculated the absorption volume from the drop volume remaining on the surface using the spherical cap approximation. Such unsaturated behavior deviation from Washburn's law was also found in several numerical studies [Reis et al. 2004, Markicevic et al 2009, Navaz et al . 2008, Willis et al. 2011]. Markicevic and Navaz [2010] showed numerically the transition from fully to partially saturated flow during droplet absorption.

For a better understanding of the absorption process inside porous media, observing directly the liquid content redistribution in the porous medium is required. Absorption in porous media has been studied with several non-destructive techniques, namely X-ray, neutron and gamma-ray radiography, 
magnetic resonance imaging (MRI) and nuclear magnetic resonance, and destructive techniques. Reis et al. [2003, 2006] used MRI measurement (of temporal resolution 3.2 minutes and spatial resolution $78 \mu \mathrm{m})$ to investigate the evaporation of droplets in glass beads substrates. They showed that the general shape of liquid redistributed within the porous media resembles a half spheroid and is comparable to CFD numerical simulation results [Reis 2003, 2004]. Jung et al. [2012] observed the absorption of impinging droplet with X-ray radiography (with temporal resolution $6.4 \mathrm{~s}$ and spatial resolution $9 \mu \mathrm{m}$ ). They documented the migration of the moisture front inside packed sand samples and distinguished two water migration regions: inertia influence region and inertia non-influence region based on velocity of the moisture front. D'Onofrio et al. [2010] measured the penetration depth of a nerve warfare agent (VX) sessile droplet into sand using a container of varying depth with detector paper on its bottom to measure the time when the liquid reaches the bottom. They showed a good agreement between numerical simulation using continuum model and their experiments. However, the above-mentioned MRI or X-ray studies had low temporal and spatial resolutions to fully capture the absorption process of a droplet in a porous medium.

In this study, we aim to capture the full absorption process of an impinging droplet on natural porous stones by using high-speed imaging, neutron radiography and numerical simulation. Neutron radiography allows visualizing the absorption process inside porous stones. Given neutron radiography limited temporal resolution, we complement this non-destructive technique with highspeed camera measurements for determining the remaining volume on the surface and thus the absorbed fraction. In addition, absorption is studied with finite-element modeling to evaluate the moisture content distribution inside the porous stone. Therefore, in contrast to previous studies, the absorption process of an impinging droplet is continuously characterized during spreading, absorption, evaporation and redistribution, in terms of absorption mass and moisture distribution, in a time-resolved manner.

\section{Materials and methods}

Porous stone samples and characteristics

Three natural porous stones are selected for the droplet impact experiments: Savonnières, Meule and Pietra serena. Savonnières is a highly porous limestone used historically in building façades (e.g. railway station Gare de l'Est, Paris or sculptures of cathedrals of Aachen and Cologne) and thus still in use for restoration purposes [Derluyn 2014, Dreesen and Dusar 2004]. The Meule sandstone (grès à meules) is composed of quartz grains ( $74 \%)$, clay and other secondary mineralization constituents and has also been used as a building material (e.g. tower of Strasbourg cathedral) [Moonen 2009]. Pietra serena is a fine-grained and compact sandstone used for building details (e.g. decorative elements of Pazzi and Medici Chapels, Florence). Figure 1 shows microscopic images of the three stone surfaces. 
The porous stones are cut in cubes of $20 \times 20 \times 20 \mathrm{~mm}^{3}$ for characterization and drop impact tests. The properties characterized are the bulk density $\rho_{\text {bulk }}$ defined as the ratio of the dry mass to the total volume, the open porosity $\Phi$ defined as the ratio of the volume of open pores to the total volume and the saturated water content $w_{\text {sat }}$ defined as the ratio of the total mass of water filling the open pore space to total volume. Also, the water absorption coefficient $A_{\text {cap }}$ is determined by measuring the absorption mass rate per unit surface in a free water uptake experiment. The capillary water content $w_{\text {cap }}$ equals the water content when the water front reaches the top of the sample during capillary absorption.

The three stones are selected in order to understand the influence of porosity and uptake characteristics on the dynamics of droplet spreading and absorption. The measured properties of the three stones are summarized in Table 1. Savonnières shows the highest porosity, fastest water absorption and largest capillary water content compared to the other stones. The capillary water content is smaller than the saturated water content due to air entrapment during imbibition from a free water surface. Pietra serena shows the lowest porosity, water uptake coefficient and capillary water content. The fraction of the pore space filled by water in the capillary saturated state in Savonnières, Meule and Pietra serena is respectively $56 \%, 72 \%$ and $80 \%$. More details on pore structure and moisture and mechanical properties of Savonnières and Meule can be found in [Derluyn 2014, Moonen 2009, Roels et al. 2003]

In addition, surface properties characterized by a contact profilometer (Surftest-211, Mitutoyo) equipped with a $5 \mu \mathrm{m}$ radius diamond-tipped stylus are found in Table 1 . The arithmetic average roughness $R_{a}$ for Savonnières, Meule and Pietra serena is respectively 10, 9 and $4 \mu \mathrm{m}$, showing the porous materials are quite rough due to the sawing process. It was not possible to measure the equilibrium contact angle due to the immediate spreading of the sessile droplet and its capillary uptake by the substrate, so no equilibrium could be found. The main components of the stones show a very low contact angle (calcite $\sim 0^{\circ}$ and quartz $\sim 11$ to $19^{\circ}$ ) indicating they are almost perfectly wetting [Ardebrant and Pugh 1991, Okayama et al. 1997, Rodriguez-Valverde et al. 2002].

The samples are conditioned at a relative humidity of $10 \% \mathrm{RH}$ and a temperature of $25^{\circ} \mathrm{C}$ before impact. 
(a)

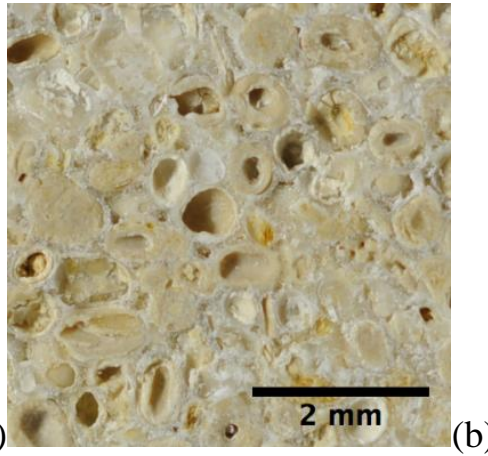

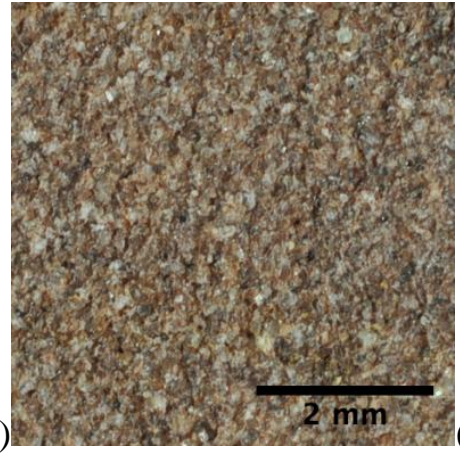

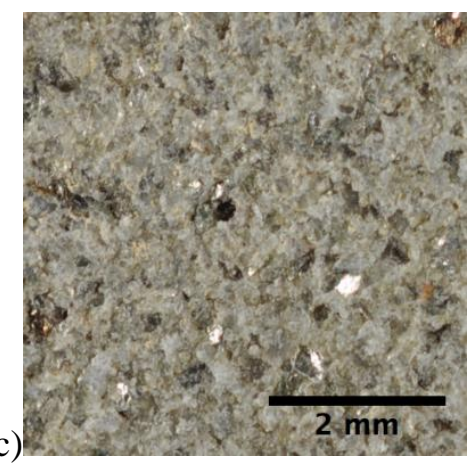

Figure 1: Microscope images for porous stones: (a) Savonnières, (b) Meule and (c) Pietra serena.

Table 1. Properties of porous stones used in the experiment

\begin{tabular}{lrrr}
\hline & Savonnières & Meule & Pietra serena \\
\hline$\rho_{\text {bulk }}\left(\mathrm{kg} / \mathrm{m}^{3}\right)$ & $1974.5 \pm 38.7$ & $2253.2 \pm 14.3$ & $2558.7 \pm 21.9$ \\
$\Phi(\%)$ & $26.9 \pm 1.4$ & $16.6 \pm 0.3$ & $5.1 \pm 0.6$ \\
$w_{\text {sat }}\left(\mathrm{kg} / \mathrm{m}^{3}\right)$ & $268.2 \pm 13.6$ & $165.7 \pm 4.0$ & $50.9 \pm 6.0$ \\
$w_{\text {cap }}\left(\mathrm{kg} / \mathrm{m}^{3}\right)$ & $151.1 \pm 6.4$ & $119.8 \pm 4.0$ & $41.6 \pm 2.5$ \\
$A_{\text {cap }}\left(\mathrm{kg} / \mathrm{m}^{2} \mathrm{~s}^{1 / 2}\right)$ & $0.089 \pm 0.012$ & $0.028 \pm 0.003$ & $0.004 \pm 0.001$ \\
$R_{a}(\mu \mathrm{m})$ & $10.3 \pm 3.5$ & $9.1 \pm 1.5$ & $4.4 \pm 1.5$ \\
\hline
\end{tabular}

\section{Droplet generation}

For the impact measurements, a water droplet is generated at the flattened tip of a needle by pushing a syringe pump. The droplet has an initial diameter $D_{0}$, reproducible with a relative error of $1 \%$. When the droplet is released, it accelerates by gravity reaching an impact velocity $V_{\mathrm{i}}$. Given the limited time at the neutron source, we only considered three impact velocities, chosen to cover the range of behavior of spreading at $0.5 \mathrm{~m} / \mathrm{s}$ and $1.0 \mathrm{~m} / \mathrm{s}$ until initiation of splashing at $3.0 \mathrm{~m} / \mathrm{s}$ as shown in Figure 2. In the high-speed camera study, the highest velocity considered is $2.0 \mathrm{~m} / \mathrm{s}$, which allows the use of a spherical cap assumption to determine the absorbed mass fraction, described next. Table 2 shows the properties of water and the impact conditions of the droplet impact tests during neutron radiography. 

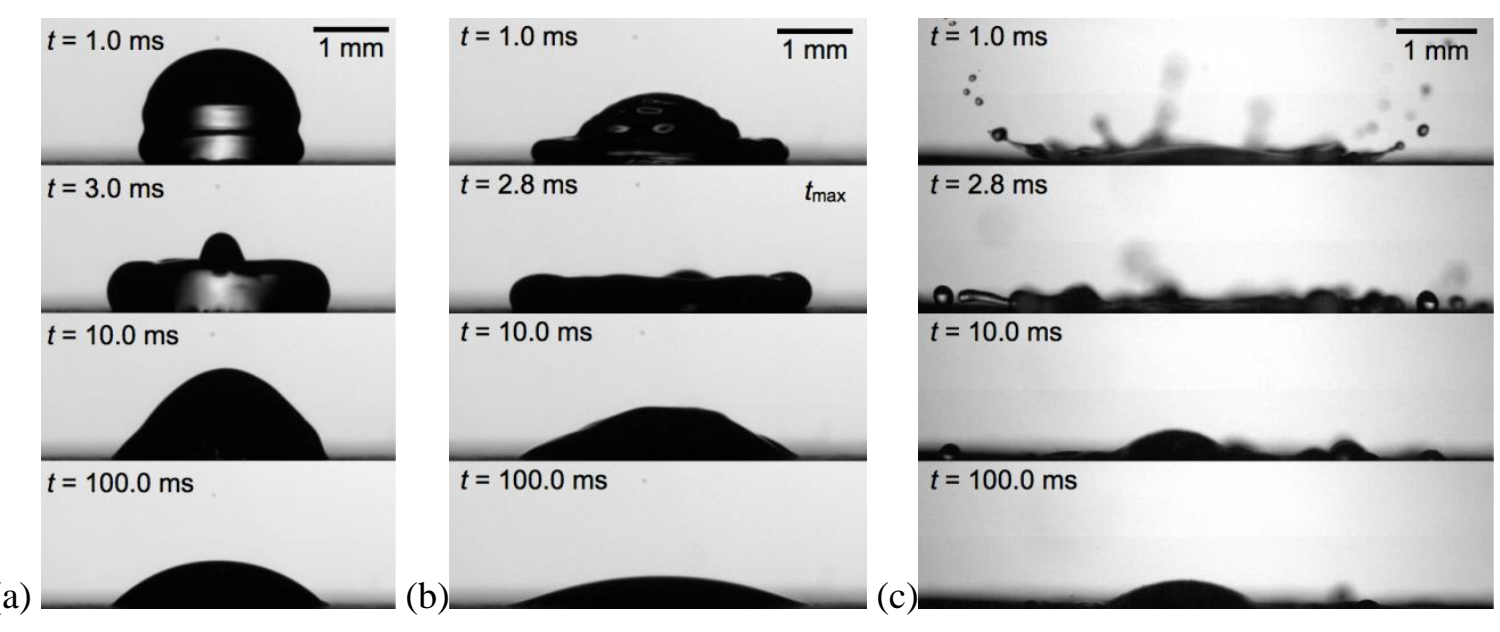

Figure 2: Drop impact on Savonnières limestone for (a) $V_{\mathrm{i}}=0.5 \mathrm{~m} / \mathrm{s}$, (b) $V_{\mathrm{i}}=1.0 \mathrm{~m} / \mathrm{s}$ and (c) $V_{\mathrm{i}}=3.0$ $\mathrm{m} / \mathrm{s}$. Deposition ( $\mathrm{a}, \mathrm{b})$ and splashing (c) can be observed.

Table 2. Properties of water droplet and impact conditions used for droplet neutron radiography

\begin{tabular}{|c|c|c|c|c|c|c|c|c|}
\hline & \multicolumn{3}{|c|}{ Water at $25^{\circ} \mathrm{C}$} & \multicolumn{5}{|c|}{ Impact condition } \\
\hline & $\begin{array}{c}\rho \\
\left(\mathrm{kg} / \mathrm{m}^{3}\right)\end{array}$ & $\begin{array}{c}\eta \\
(\mathrm{mPa} \cdot \mathrm{s})\end{array}$ & $\begin{array}{c}\gamma \\
(\mathrm{mN} / \mathrm{m})\end{array}$ & $\begin{array}{c}D_{0} \\
(\mathrm{~mm})\end{array}$ & $\begin{array}{c}m \\
(\mathrm{mg})\end{array}$ & $\begin{array}{c}V_{\mathrm{i}} \\
(\mathrm{m} / \mathrm{s})\end{array}$ & We & $\operatorname{Re}$ \\
\hline Water & 998 & 1.0 & 72.8 & 2.0 & 4.3 & $\begin{array}{l}0.5 \\
1.0 \\
3.0\end{array}$ & $\begin{array}{c}7 \\
27 \\
247\end{array}$ & $\begin{array}{c}998 \\
1996 \\
5988\end{array}$ \\
\hline
\end{tabular}

\section{High-speed camera imaging}

The drop impact on the porous surface is captured with shadowgraphy images using a high-speed camera setup (1 $000 \mathrm{fps}, 7.38 \mu \mathrm{m}$ spatial resolution and $30 \mu$ s exposure time) in order to measure the depletion of the droplet and the mass fraction taken up by the porous medium. The frame rate is optimized such that the full absorption process can be captured considering the memory limitations of the high-speed camera. The drop volume on the surface is determined from the side view images. We assume the droplet on the surface to have a spherical cap shape. The height of the drop apex $h(t)$, the width of the droplet base $D_{C L}(t)$ and the radius $R(t)$, as depicted in Figure 3, are determined from the image using a custom made MATLAB code. Using these data as input, the drop volume on surface and the contact angle $\theta_{s c}(t)$ can be determined as

$$
\begin{gathered}
V_{\text {drop }}=\frac{\pi h(t)}{6}\left(3\left(\frac{D_{C L}(t)}{2}\right)^{2}+h(t)^{2}\right) \\
\theta_{s c}(t)=\arctan \frac{D_{C L}(t) / 2}{R(t)-h(t)}
\end{gathered}
$$

The absorption mass $M_{a b s}$ is given by:

$$
M_{\mathrm{abs}}=M_{0}-\rho \cdot V_{\mathrm{drop}}
$$

where $M_{0}$ is the mass of the initial droplet. The initial mass $M_{0}$ is controlled by the injection system and equals $M_{\text {drop }}=4.3 \mathrm{mg}$ with a systematic error of less than $2 \%$. The evaporation of a drop 
deposited on a nonporous surface leads to a volume loss of less than $3 \%$ in 10 seconds. Thus evaporation is found to be negligible for these droplet depletion investigations, where depletion times are less than 10 seconds. We also evaluated the accuracy of the spherical cap approach for determining the remaining volume $V_{\text {drop }}$ by depositing a droplet on an impermeable surface. The global systematic error was found to be less than 3\%. When the droplet becomes very flat at the end of the depletion process, the method is not able to measure the volume precisely, due to inaccurate measurement of drop height, caused by reflection from the liquid puddle and bubble eruption.

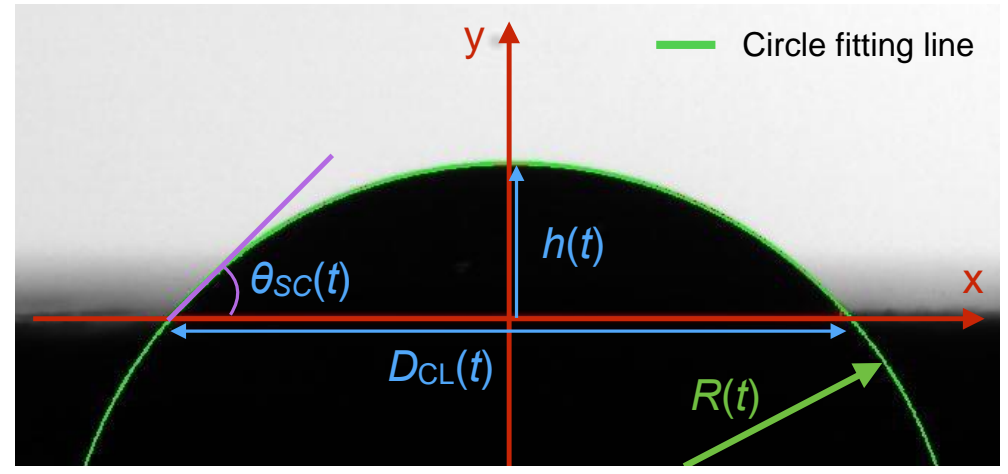

Figure 3: Illustration of the spherical cap description for droplet deposited on porous medium.

Neutron radiography

The absorption process into the porous stone is captured by neutron radiography. Neutrons are attenuated by the hydrogen of water, but penetrate the porous stone. The experiment for the absorption of drop impact is performed at the NEUtron Transmission RAdiography (NEUTRA) beamline of the Paul Scherrer Institut, Villigen, Switzerland. The NEUTRA beamline is operated with neutrons within a thermal spectrum [Lehmann 2008]. Figure 4 shows a schematic overview of the neutron beamline and the experimental setup for drop impact. The steady state spallation-neutron source, driven by a $590 \mathrm{MeV}$ proton beam from a ring cyclotron with a power in the MW range produces $25 \mathrm{meV}$ neutrons in a heavy water moderator tank from which they are extracted through the collimator. The transmitted neutrons through the sample are converted into visible light photons by the scintillator. The scintillator is a mixture of $\mathrm{ZnS}$ crystals and a binder, doped with ${ }^{6} \mathrm{Li}$ as neutron absorbing agent (200 $\mu \mathrm{m}$ thick). The photons are then led via a mirror onto a CMOS camera Andor Neo. The necessary exposure time for each image is 3 seconds and the nominal spatial resolution of the neutron radiography is $45.5 \mu \mathrm{m} / \mathrm{pixel}$. 


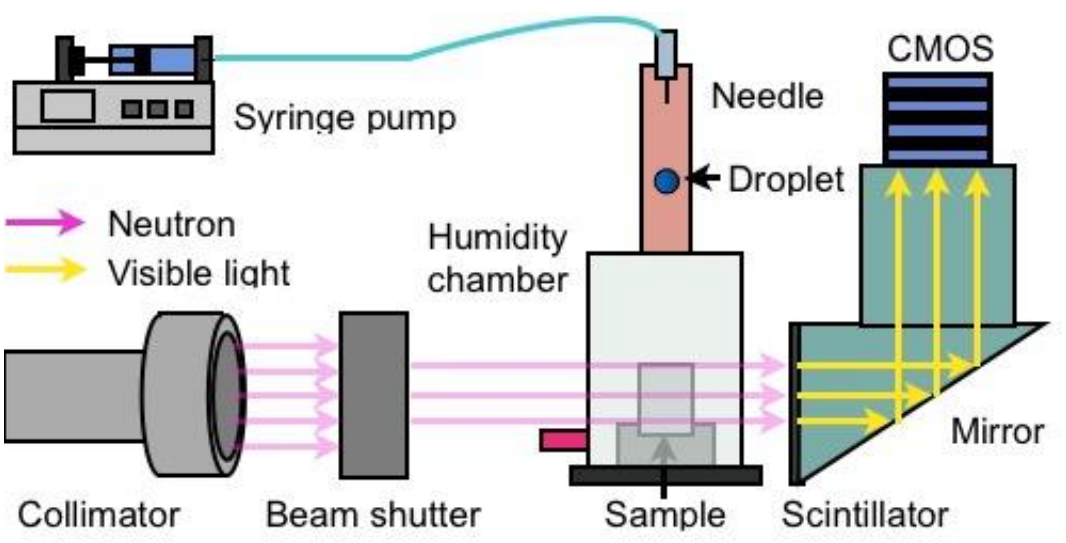

Figure 4: Schematic of NEUTRA beamline configuration.

The captured neutron radiographs are quantified using a custom MATLAB code. To improve the quality of neutron radiographs and to quantify the transmission value, several corrections (dark field, flat field and intensity) are applied to the images. The dark field correction compensates for the background noise of the CMOS camera. The flat field correction eliminates inhomogeneities of the beam and detector by compensating for different gains and dark currents. The intensity correction scales the measured radiograph, so that the same neutron source flux is assumed for all radiographs of a series, more details are found in [Sedighi Gilani et al. 2012].

The corrected transmission value $T^{\prime}$ on each pixel is obtained with the monochromatic exponential attenuation law (Beer-Lambert), expressed as:

$$
T^{\prime}(t)=\frac{I}{I_{0}}=\exp (-\Sigma \cdot d(t))
$$

where $T^{\prime}(t)$ is the change of the transmission based on the time $t, I_{0}$ is the intensity of the incident beam, $I$ is the intensity of the transmitted beam, $d(t)$ is the total thickness of the object in the beam direction and $\Sigma$ is the linear attenuation coefficient. After drop impact, the sample can be thought of as a layer of porous medium (stone) with empty pores and constant thickness $d_{\text {stone, }}$, plus a layer of water with thickness $d_{\text {water }}(t)$ [Sedighi Gilani et al. 2012]. The transmission value of the neutron radiography after drop impact can be described as:

$$
T^{\prime}{ }_{\text {drop }}(t)=\exp \left(-\Sigma_{\text {stone }} \cdot d_{\text {stone }}-\Sigma_{\text {water }} \cdot d_{\text {water }}(t)\right)
$$

For a radiography of the initially dry sample, the transmission value can be written as:

$$
T_{\text {stone }}^{\prime}=\exp \left(-\Sigma_{\text {stone }} \cdot d_{\text {stone }}\right)
$$

and then the water mass $M(t)$ is:

$$
M(t)=\frac{\rho \cdot \text { pixelsize }^{2}}{\Sigma_{\text {water }}} \ln \left(\frac{\mathrm{T}_{\text {stone }}^{\prime}}{\mathrm{T}_{\text {drop }}^{\prime}(t)}\right)
$$


where the water attenuation coefficient is $\Sigma_{\text {water }}=3.63(1 / \mathrm{cm})$. The water mass calculated from the neutron radiography taken at $3 \mathrm{~s}$ after drop impact is compared with the water mass given by drop injection system, and less than $6.5 \%$ difference is found.

\section{Experimental results}

\section{Droplet behavior}

We first look at the high-speed camera results to examine qualitatively the behavior of the droplet. We present examples of high-speed camera snapshots of drops impacting Savonnières at different velocities in Figure 2. At impact velocities $V_{\mathrm{i}}=0.5$ (Fig.2a) and $V_{\mathrm{i}}=1.0 \mathrm{~m} / \mathrm{s}$ (Fig.2b), the liquid droplet spreads over the porous surface until it reaches its maximum spreading diameter $(t \approx 0.003 \mathrm{~s})$. After maximum spreading, the droplet shows some dynamic behavior, but remains pinned at its edges $(t=0.01 \mathrm{~s})$, as mentioned above [Lee et al. 2015]. Once the kinetic energy of drop impact is dissipated, the droplet forms a spherical cap on the surface $(t=0.1 \mathrm{~s})$. The droplet does not attain an equilibrium state since capillary absorption into the substrate occurs. At high impact velocity at $V_{\mathrm{i}}=$ $3.0 \mathrm{~m} / \mathrm{s}$ (Fig. 2c), the drop splashes and different droplet fragments are spread over the contact area, forming themselves satellite spherical cap droplets, from where absorption takes place.

The contact line diameter, $D_{\mathrm{CL}}$, and the dynamic contact angle, $\theta_{\mathrm{D}}$, measured on the high-speed images are plotted versus time in Figure 5. In terms of contact line diameter, Pietra serena attains in 3 ms a slightly higher maximum spreading than Savonnières and Meule do in about $2 \mathrm{~ms}$. After this initial spreading, the contact line diameter $D_{\mathrm{CL}}$ remains constant for all three stones, confirming that the droplet is pinned. Looking at the dynamic contact angle on the different porous stones, we see that the contact angle reaches a maximum during the spreading phase, then decreases while staying pinned and afterwards oscillates when reaching its equilibrium value which brings us at $10 \mathrm{~ms}$ from impact. The dynamic contact angle during spreading on all three stones shows a dynamic non-wetting or hydrophobic behavior, with a contact angle $\theta_{\mathrm{D}}>90^{\circ}$. After this maximum spreading, the dynamic contact angle shows a dynamic wetting behavior, with a contact angle $\theta_{\mathrm{D}}$ between 20 and $60^{\circ}$. It is noted that, after initial spreading, the dynamic contact angle is in line with the behavior of the porous stones observed in quasi-static conditions, where the samples are hydrophilic as indicated by their capillary active behavior. A simple explanation of the hydrophobic behavior during spreading $\left(\theta_{\mathrm{D}}>\right.$ $90^{\circ}$ ) could be the presence of air entrapped in the surface pore structure forming a thin air layer between the liquid and the solid material. We will come back on this issue in the discussion section. 

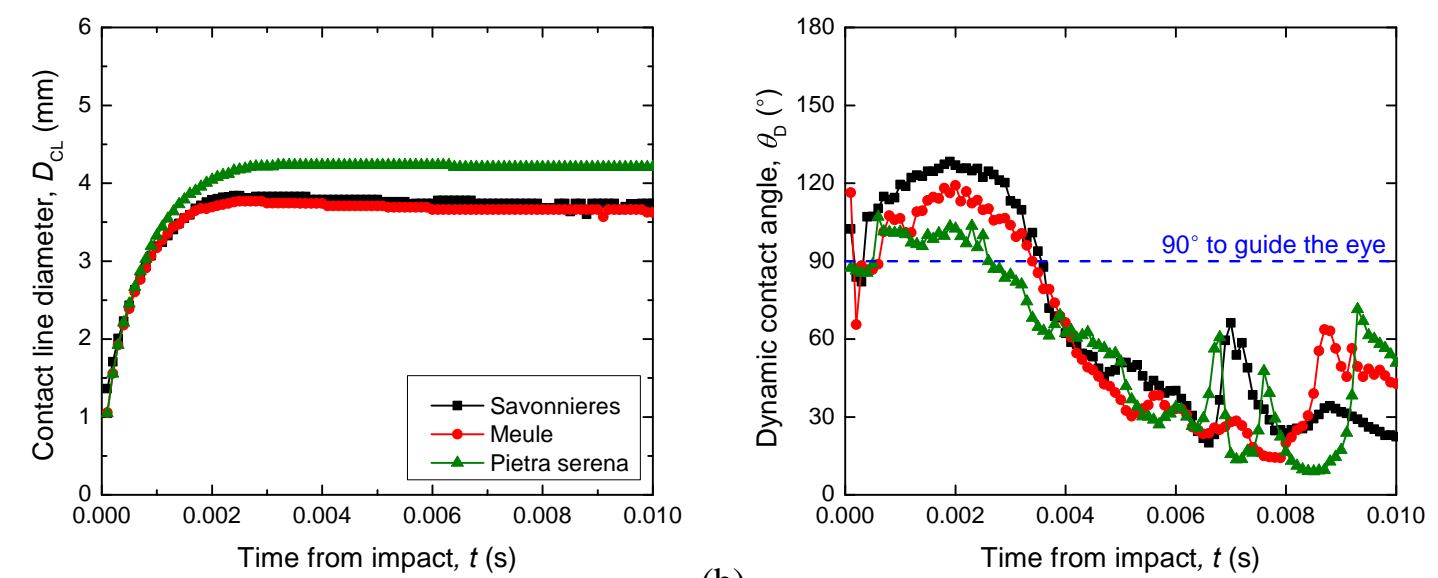

(a)

(b)

Figure 5: First $10 \mathrm{~ms}$ of droplet impact: a) contact line diameter, $D_{\mathrm{CL}}$, and b) dynamic contact angle, $\theta_{\mathrm{D}}$, versus time for $D_{0}=2.0 \mathrm{~mm}$ and $V_{\mathrm{i}} \sim 1.0 \mathrm{~m} / \mathrm{s}$ on porous stones [Lee et al.2015].

We now consider the droplet behavior after this initial short time period where the impact energy is dissipated. Figure 6 shows the time evolution of the contact line diameter $D_{\mathrm{CL}}$ and contact angle $\theta_{s c}$ during this capillary absorption phase from 0.1 to 10 seconds after impact for the three porous materials and the three impact velocities. Comparing the contact line diameter at 0.1 second, or at the onset of the absorption process, this diameter is higher for higher impact velocity. In reverse, the contact angle at the start of the absorption process decreases with impact velocity, which is logical since a spherical cap with the same mass but wider diameter shows a lower contact angle. The quasistatic contact angles during the absorption phase are remarkably smaller $\left(\theta_{\mathrm{D}} \sim 20-80^{\circ}\right)$ than the dynamic contact angles at maximum spreading.

For Savonnières and Meule, the contact line diameter $D_{\mathrm{CL}}$ remains constant during a certain time, while the contact angle decreases from start as water transfers from the droplet into the porous stone by capillary absorption. This type of behavior is called the CCR (constant contact radius) mode Marmur [1988].The CCR mode is schematically illustrated in Figure 7a and captured by snapshots for Meule in Figure $7 \mathrm{~b}$. At lower impact velocity $\left(V_{\mathrm{i}}=0.5\right.$ and $\left.1 \mathrm{~m} / \mathrm{s}\right)$, the CCR mode continues almost until droplet depletion. For high impact velocity $\left(V_{\mathrm{i}}=2 \mathrm{~m} / \mathrm{s}\right)$, the droplet starts to deplete much earlier, since the droplet is spread over a larger area with smaller droplet height. The decreasing contact diameter is in this case is not due to a depinning of the contact line, but due to the borders of the droplet becoming totally absorbed. It is to be remarked that a very fast depletion of the droplet is incurred at a contact angle of about $10^{\circ}$ for all impact velocities, which is close to the equilibrium contact angle. Figure 6e-f shows that, for Pietra serena, the contact line diameter and contact angle remain almost constant through the experiment, which is explained by the very slow capillary absorption by this material. 
(a)
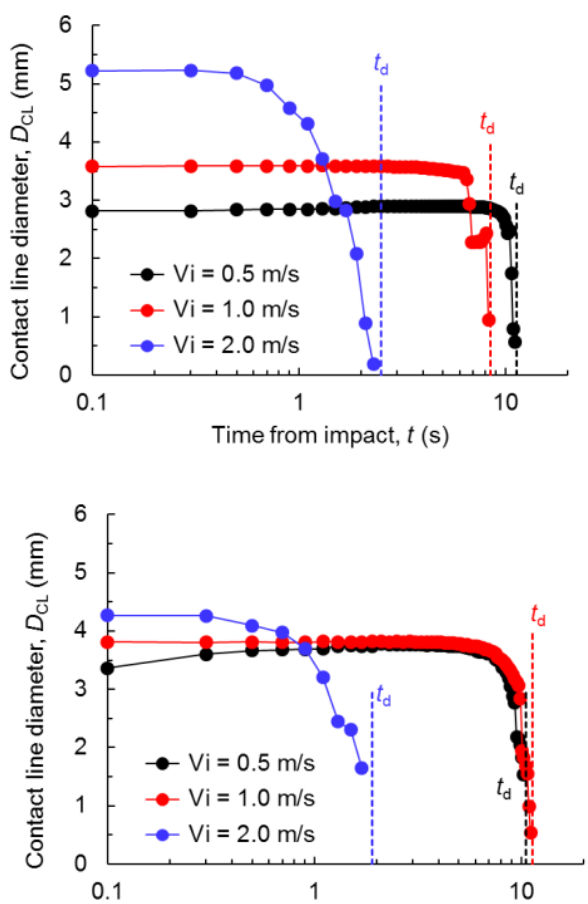

(c)

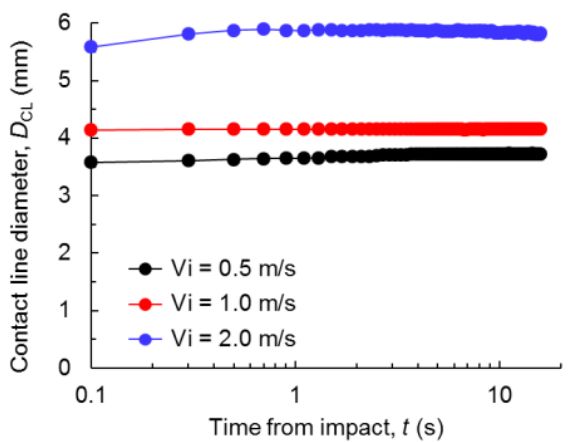

(b)

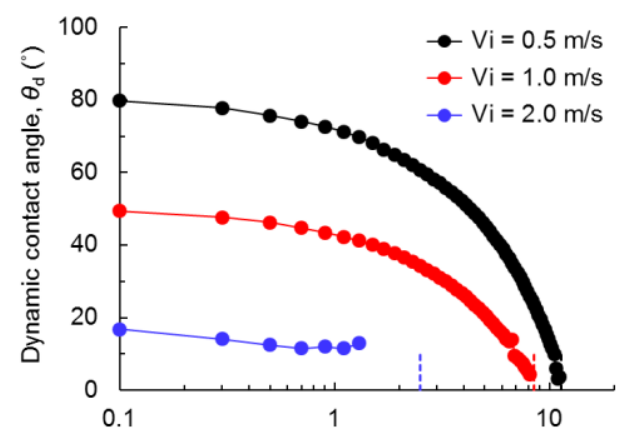

Time from impact, $t$ (s)

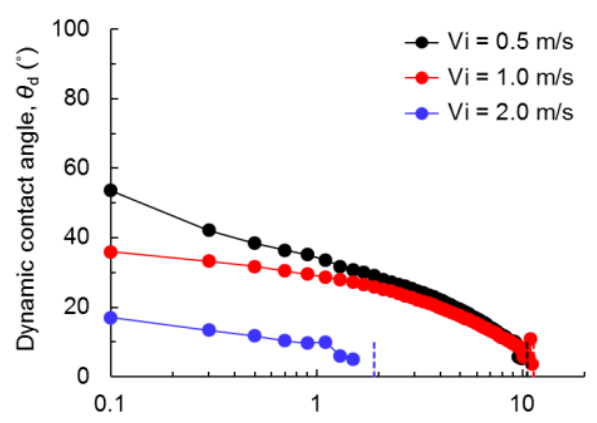

(d)

Time from impact, $t$ (s)

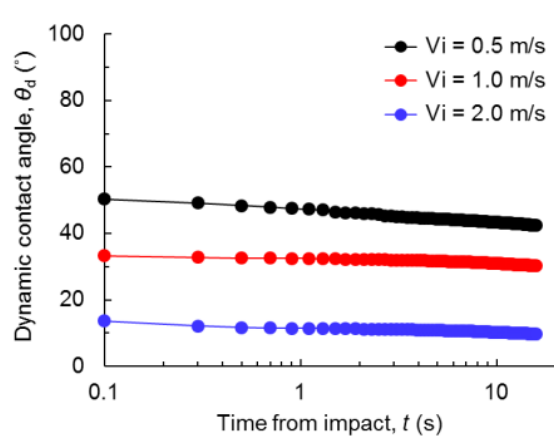

(f)

Figure 6: Time evolution of contact line diameter $D_{\mathrm{CL}}$ and contact angle $\theta_{\mathrm{sc}}$ in absorption phase (a-b) Savonnières, (c-d) Meule and (e-f) Pietra serena. The dashed vertical lines indicate the depletion time $\left(t_{\mathrm{d}}\right)$.

(a)

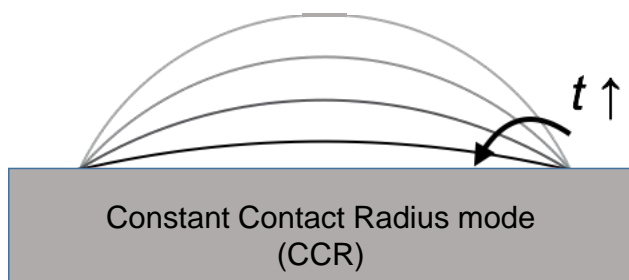

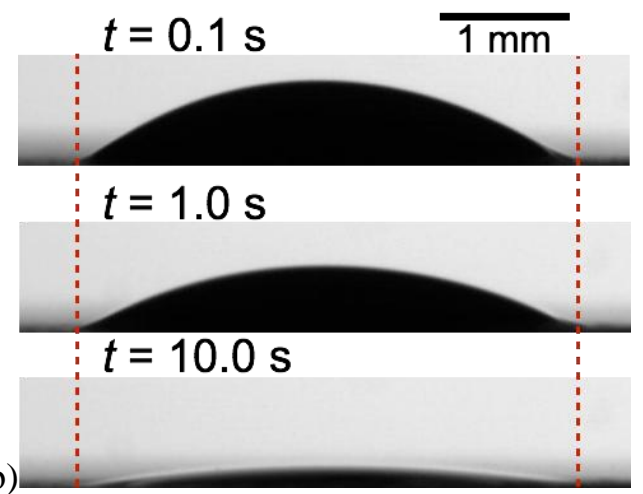

Figure 7: (a) Schematic of absorption with constant contact radius (CCR) and (b) snapshots of remaining droplet $\left(V_{\mathrm{i}}=1.0 \mathrm{~m} / \mathrm{s}\right)$ on Meule sandstone during absorption phase. 


\section{Moisture absorption}

Neutron radiography images the droplet and the stone at 3 -second intervals over 100 seconds. We show in Figure 8 moisture distribution at 3, 9, 18 and 30 seconds in terms of water mass ( $\mu \mathrm{g})$ per pixel $\left(45.5 \times 45.5 \mu \mathrm{m}^{2}\right)$ for the three stones and the three impact velocities. The total mass detected at the initial image is calibrated with the known mass of the water droplet, i.e. $4.3 \mathrm{mg}$. For readability, the top surface of the stone is indicated with a horizontal black line.

After spreading and pinning, the pinned droplet becomes a reservoir for water uptake and its contact area with the stone thus determines the entry path of water. At low impact velocity, the contact area is lower, so moisture uptake is slower and the droplet lasts longer on the stone, being visible at 3 and 9 seconds for Savonnières and Meule. At higher impact velocity, the droplet has high contact area and the thin reservoir is rapidly depleted, as shown in Figure 8 for Savonnières and Meule at $3.0 \mathrm{~m} / \mathrm{s}$ impact velocity. Thus, the time at which the droplet is depleted shortens with increasing impact velocity. For Pietra serena, the water uptake is much slower and the contact area is maintained even after 30 seconds as very little water has been absorbed. In the following, due to its very slow absorption behavior, Pietra serena is not further discussed in this paper and we will focus on Savonnières and Meule.

For Savonnières and Meule at time $t=18 \mathrm{~s}$ and $t=30 \mathrm{~s}$, the droplet is depleted and redistribution within the porous material and evaporation at the surface occur. While the width of the wetted zone in the porous material in horizontal direction depends on the impact velocity, the depth of the wetted zone seems to be less dependent on impact speed. We note that the width of the wetted zone, being much wider than the contact line diameter of the deposited droplet clearly indicates that the droplet becomes a point source for tri-dimensional uptake, also along the surface.

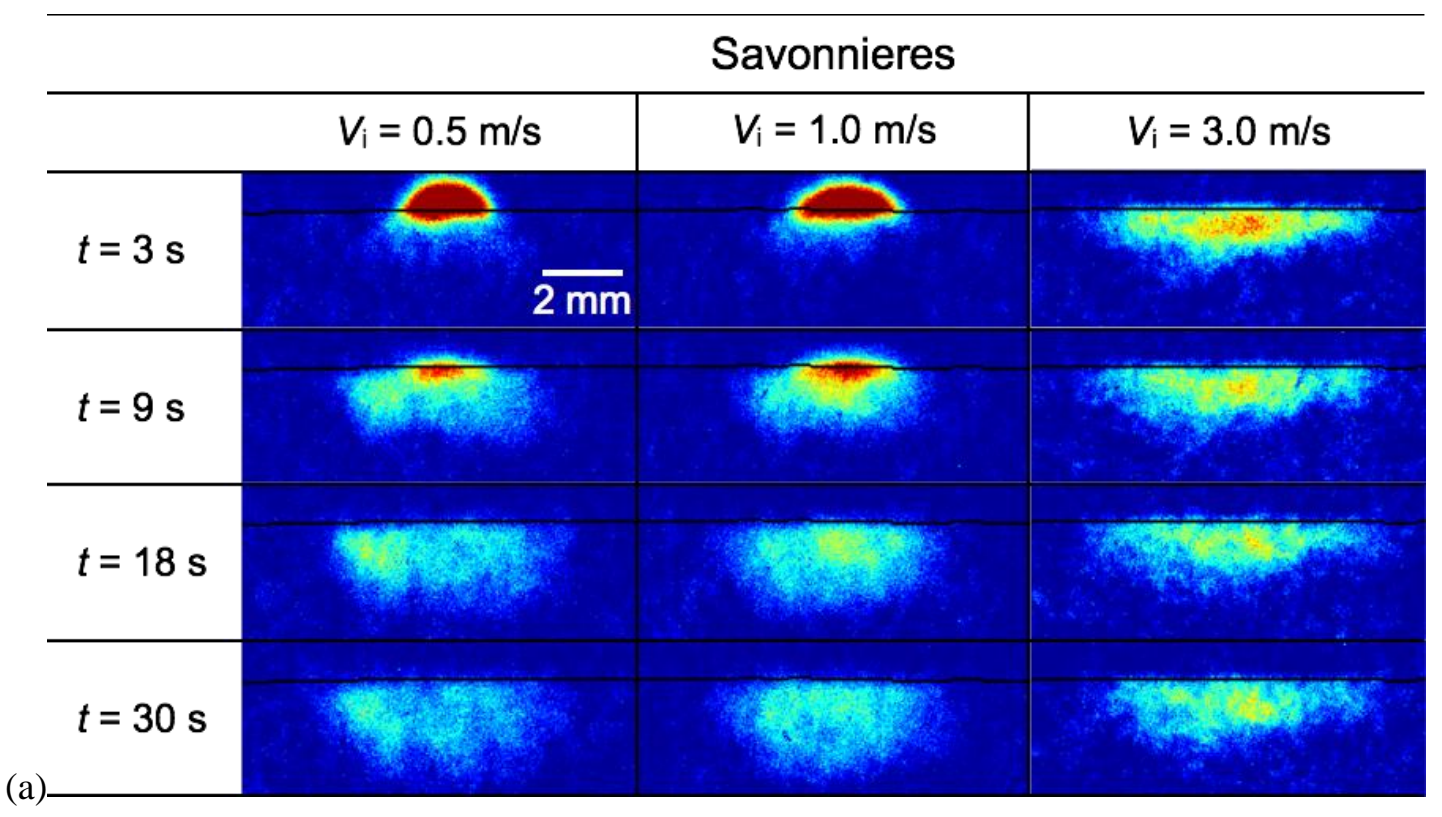



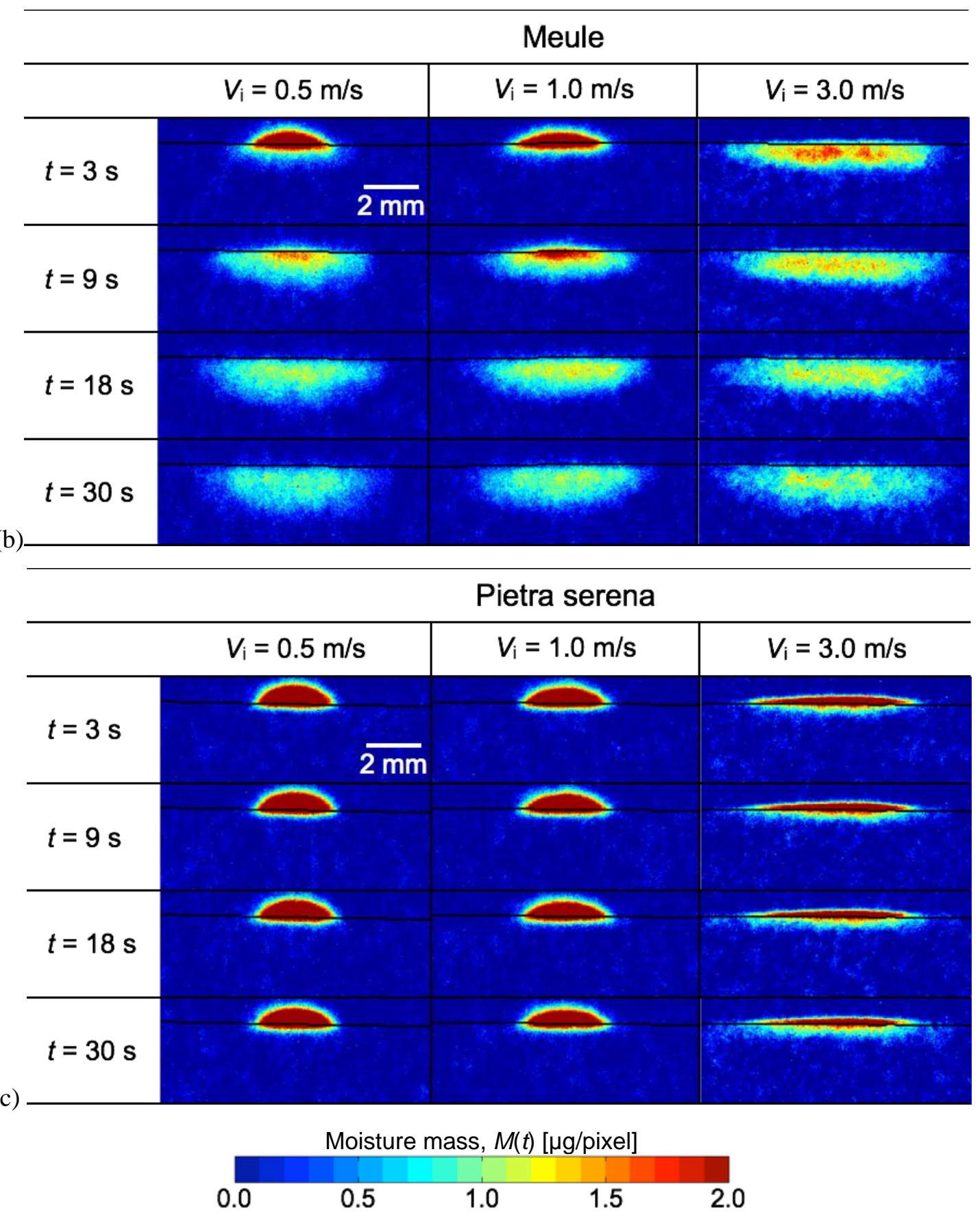

Figure 8: Snapshots of neutron radiography for moisture mass distribution of drop impact with different impact velocities in porous stones: (a) Savonnières limestone, (b) Meule sandstone and (c) Pietra serena. The sensitivity of neutron imaging to liquid water is highlighted by the moisture mass legend showing a maximum of $2 \mu \mathrm{g}$.

We remark that neutron images are obtained in projection mode, so the moisture mass at a given pixel position aggregates all the moisture along the depth of the sample. Profiles of the moisture mass at the center of the droplet are obtained from the neutron images by averaging the data over a width of 10 pixels (Fig. 9a). Figure 9b shows such mass profiles versus time for a droplet with impact velocity $V_{\mathrm{i}}=0.5 \mathrm{~m} / \mathrm{s}$ on Savonnières. The profiles above the surface refer to the droplet sitting on the surface. 
The profiles in the porous stone have initially high moisture levels, which decrease as moisture is taken up further into the porous material. When the droplet is depleted, the evaporation phase starts and the moisture mass at the surface decreases. Concurrently with evaporation, downward transport into the porous material continues, also referred to as redistribution.

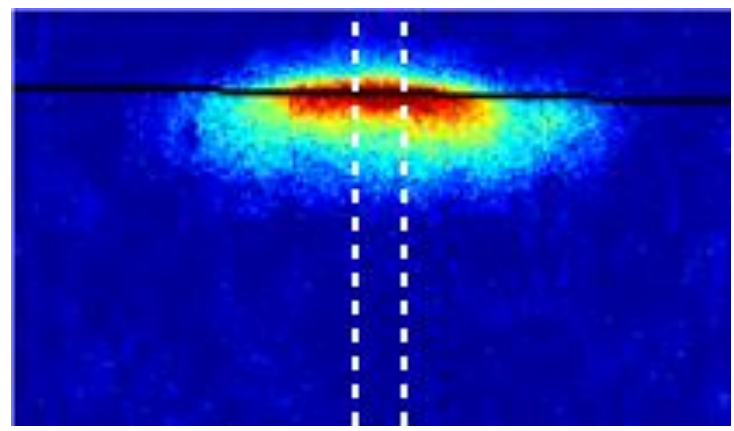

(a)

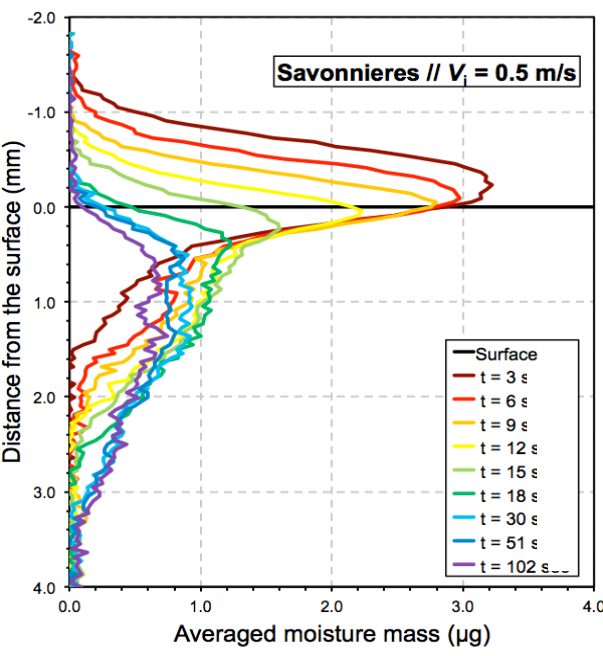

(b)

Figure 9: (a) Schematic representation of averaging the moisture mass over a width of 10 pixels at the centerline of the neutron radiagraph. (b) Averaged moisture mass profiles at the droplet with impact velocity $V_{\mathrm{i}}=0.5 \mathrm{~m} / \mathrm{s}$ on Savonnières.

Both for the high-speed images and the neutron radiographs, the total mass of moisture for each image is determined and moisture mass is plotted in function of time, as shown in Figure 10 with the absorbed mass normalized by the initial drop mass presented for Savonnières and Meule, for different impact velocities. The results show good agreement between the two measurement methods at $V_{\mathrm{i}}=$ 0.5 and $1.0 \mathrm{~m} / \mathrm{s}$ for both porous materials. The absorption phase with increasing absorbed mass and the evaporation phase where the mass decreases again can clearly be distinguished. At the end of the absorption phase, the droplet is depleted. Droplet depletion time shortens with impact speed, as seen above. To verify the likelihood of liquid penetration during impact and initial spreading, the curves are extrapolated to $t \approx 0.003 \mathrm{~s}$, i.e. the time at maximum spreading, indicating penetration of liquid during the first microseconds is unlikely to occur.

We replot the data in a log-log (Figures 10b-d), comparing the slope of the data with the 0.5 slope as predicted by the Washburn equation. While Meule could show a consistency with the Washburn law during the absorption phase, the data of Savonnières clearly deviate from Washburn law. Reason is that the absorption process is not unidimensional from an infinite water source, but tridimensionally into the porous material, as also seen in the neutron radiographs, Fig. 8. 

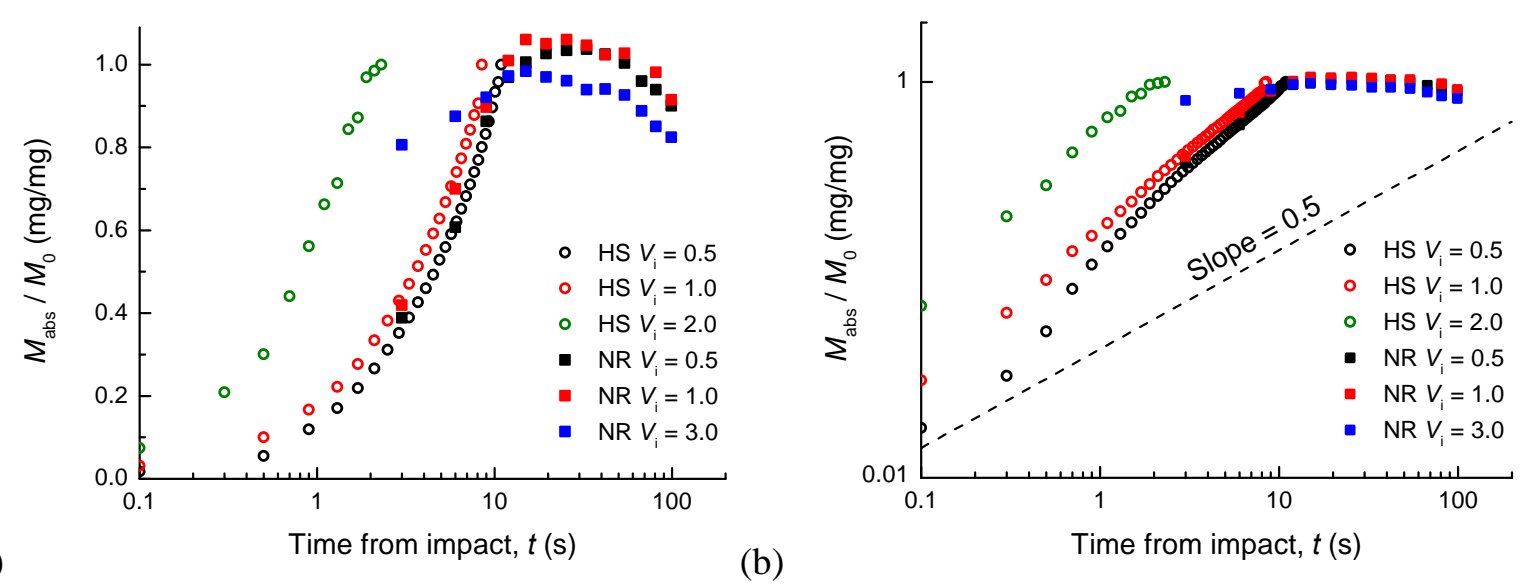

(a)

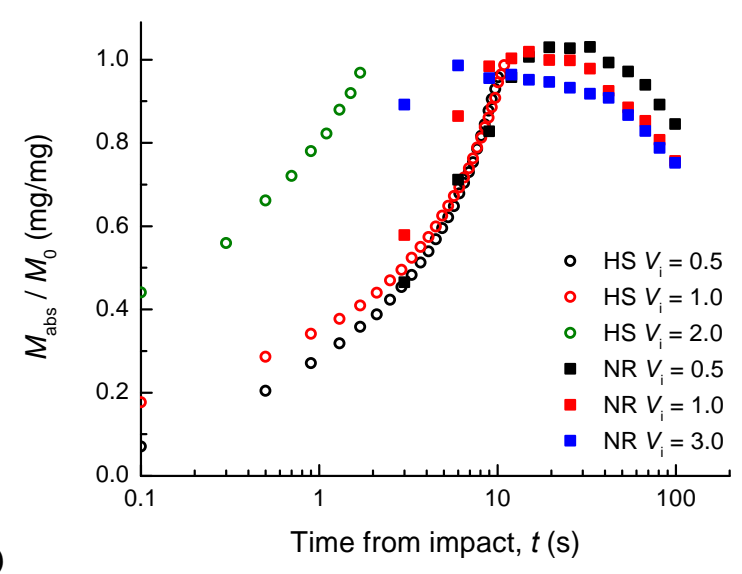

(c)

(d)

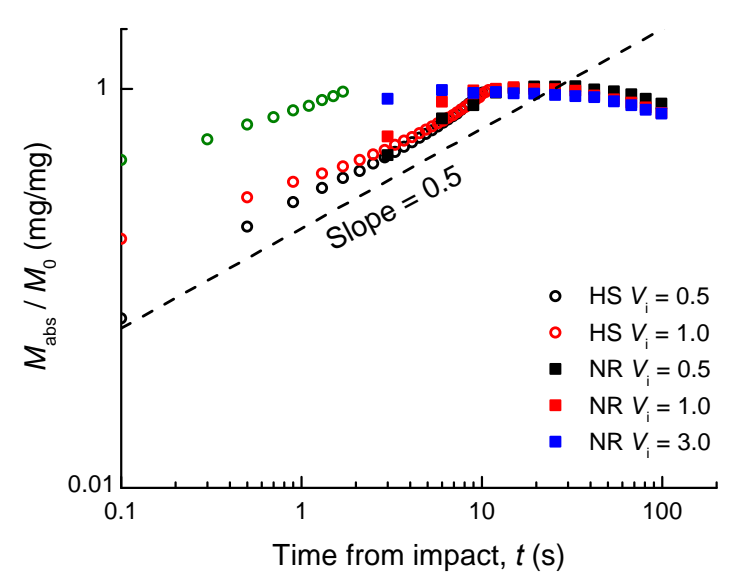

Figure 10: Normalized absorption mass versus time for (a-b) Savonnières and (c-d) Meule samples. Data extracted from the HS: high-speed images, NR: neutron radiography. Figure 10a-c are in semi$\log$ and $10 \mathrm{~b}-\mathrm{d}$ in $\log -\log$.

\section{Numerical model and results}

In this section, we present a model to describe the absorption, evaporation and redistribution processes in the porous material. Moisture absorption from the droplet, evaporation at the surface and redistribution in the porous material is modelled using a continuum approach, assuming unsaturated capillary flow in a non-deformable porous medium. Assuming a symmetric droplet shape at the surface of the porous material, a two-dimensional geometry with axial symmetry is considered as shown in Fig 11a. Since the droplet remains pinned for an important time during the absorption process, for simplicity we assume that droplet diameter is constant during the total depletion process. This assumption is valid for low impact velocities, but becomes an inferior approximation for high impact velocities. The droplet depletion is modelled with the droplet approximated to a limited reservoir assuming uniform boundary conditions at the contact area by imposing a constant capillary pressure at the interface. The droplet volume is monitored during uptake until it is emptied, i.e. until depletion of the droplet. After droplet depletion, boundary conditions are changed to model the evaporation at the surface while moisture redistribution within the material goes on. 
Isothermal moisture transport consists in liquid and vapor transport driven by a gradient in capillary pressure $p_{c}$ :

$$
\frac{\partial w}{\partial t}=\nabla \cdot\left(\left(K_{l}+\frac{\delta_{v} \cdot p_{v, \text { sat }} \cdot R H}{\rho \cdot R_{v} \cdot T}\right) \nabla p_{c}\right)
$$

with $w$ the volumetric moisture content, $K_{l}$ the liquid permeability, $\delta_{v}$ the vapor permeability, $p_{v \text {,sat }}$ the saturated vapor pressure, $R H$ the relative humidity, $R_{v}$ the specific gas constant for water vapor, $T$ the temperature [Janssen et al. 2007]. In the derivation of eqn. 7, local equilibrium between liquid and vapor phases is assumed as expressed by Kelvin's law, which relates the relative humidity to the capillary pressure:

$$
R H=\exp \left(-\frac{p_{c}}{\rho \cdot R_{v} \cdot T}\right)
$$

The material modelled is Savonnières. The wetting capillary pressure curve is described by a multimodal van Genuchten curve assuming three pore systems [Derluyn 2012]. The liquid permeability of the material is a nonlinear function of capillary pressure, $K_{l}=K_{l}\left(p_{c}\right)$ and is determined using the methodology described in [Derluyn 2012, Carmeliet and Roels 2001]. The vapor permeability $\delta_{v}(R H)$ is based on wet cup/dry cup measurements [Derluyn 2012]. Figure 11c shows the capillary pressure curve and permeability curve for Savonnières.

Initially, the entire domain is conditioned at $R H=10 \%$, equal to a capillary pressure $p_{c, t=0}=1.0 \mathrm{E} 8.5 \mathrm{~Pa}$, which in turn equals, according to the capillary pressure curve in Figure $11 \mathrm{c}$, to a moisture content $\approx 0$ $\mathrm{kg} / \mathrm{m}^{3}$. The boundary condition at the top surface of the material being in contact with the droplet, zone 1 in Fig. 11a, is modeled as a Dirichlet condition, imposing a capillary pressure $p_{c}=p_{c, d r o p}$. We assume that the moisture content at the droplet/porous material interface equals the capillary moisture content as obtained in a capillary uptake experiment from a free water surface $(w \approx 132$ $\mathrm{kg} / \mathrm{m}^{3}$ ). Thus, according to the capillary pressure curve in Fig. $11 \mathrm{c}$, this equals to a capillary pressure $p_{c, d r o p}=10 \mathrm{~Pa}$.

The boundary condition at the top surface, zone 2 during absorption phase and zones 1 and 2 during evaporation phase (Fig 11b), is of Neumann type describing the moisture exchange due to evaporation:

$$
g_{v}=\beta \cdot\left(p_{v, \text { env }}-p_{v, s}\right)
$$

where $g_{v}$ is the vapor flux, $\beta$ the mass transfer coefficient, $p_{v, e n v}$ the vapor pressure of the environment and $p_{v, s}$ the vapor pressure at the porous material surface. The mass transfer coefficient is estimated from the heat transfer coefficient using the analogy from Chilton andColburn [1934] analogy and equals $\beta=4 \mathrm{E}-8 \mathrm{~s} / \mathrm{m}$. The temperature and $\mathrm{RH}$ of the surrounding air is assumed to be constant, $T=25^{\circ} \mathrm{C}$ and $\mathrm{RH}=10 \%$.

On the lateral and bottom sides (zones 3 and 4) and on the symmetry axis (zone 5 in Fig. 11b) zero flux conditions are imposed, $g_{v}=0$. 
The governing equations are solved using the finite element method in COMSOL Multiphysics (v5.0, Comsol Inc.). The mesh consists of rectangular elements, with a refinement towards the surface of the sample, where steep moisture gradients are expected to occur (Fig. 11b). The total number of elements is 150 x 150 (22 500 elements), where the smallest element height is $15 \mathrm{E}-6 \mathrm{~m}$. Adaptive time stepping is used, with an initial time step of $10 \mathrm{E}-6 \mathrm{~s}$ and a maximum time step set to $0.01 \mathrm{~s}$ during the absorption phase.

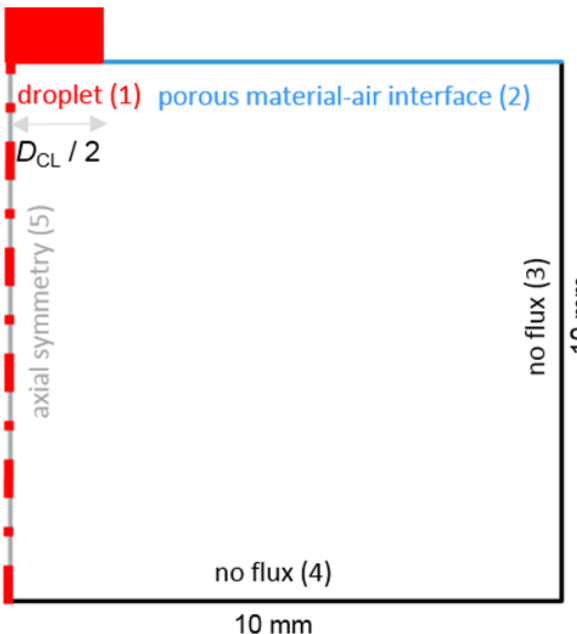

(a)

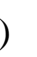

$10 \mathrm{~mm}$

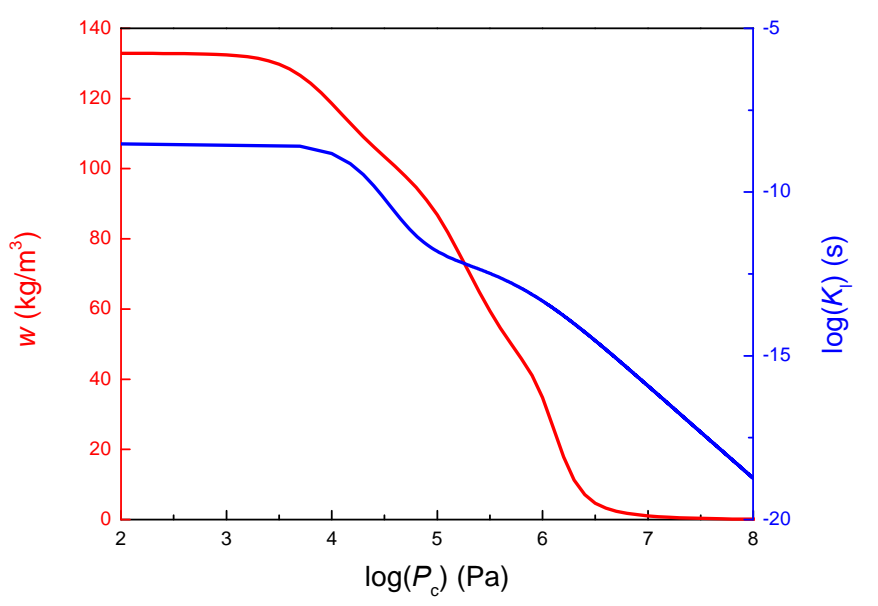

c)

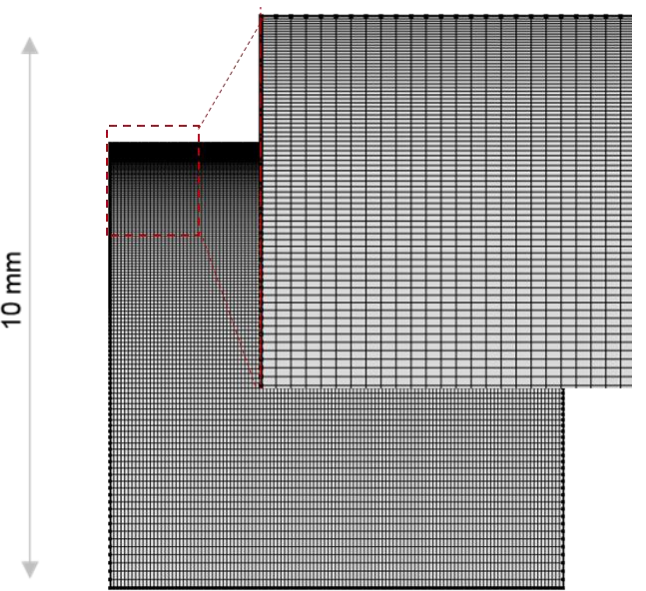

b)

Figure 11: (a) Schematic of model geometry and boundary conditions. (b) Illustration of finite element mesh. The insertion shows a magnified view close to the sample surface, with decreasing element size towards the top surface. (c) Capillary pressure and permeability curves for Savonnières limestone. 

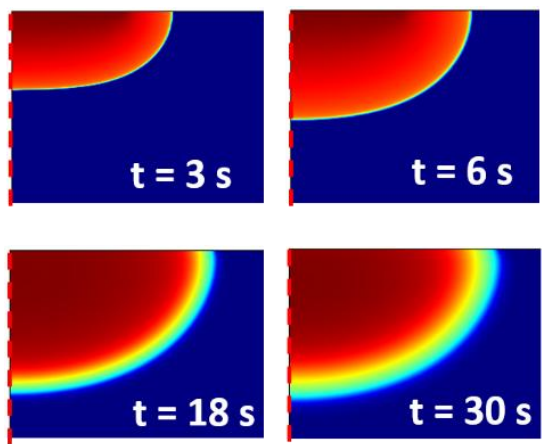

Moisture content $\left[\mathrm{kg} / \mathrm{m}^{3}\right]$

(a)
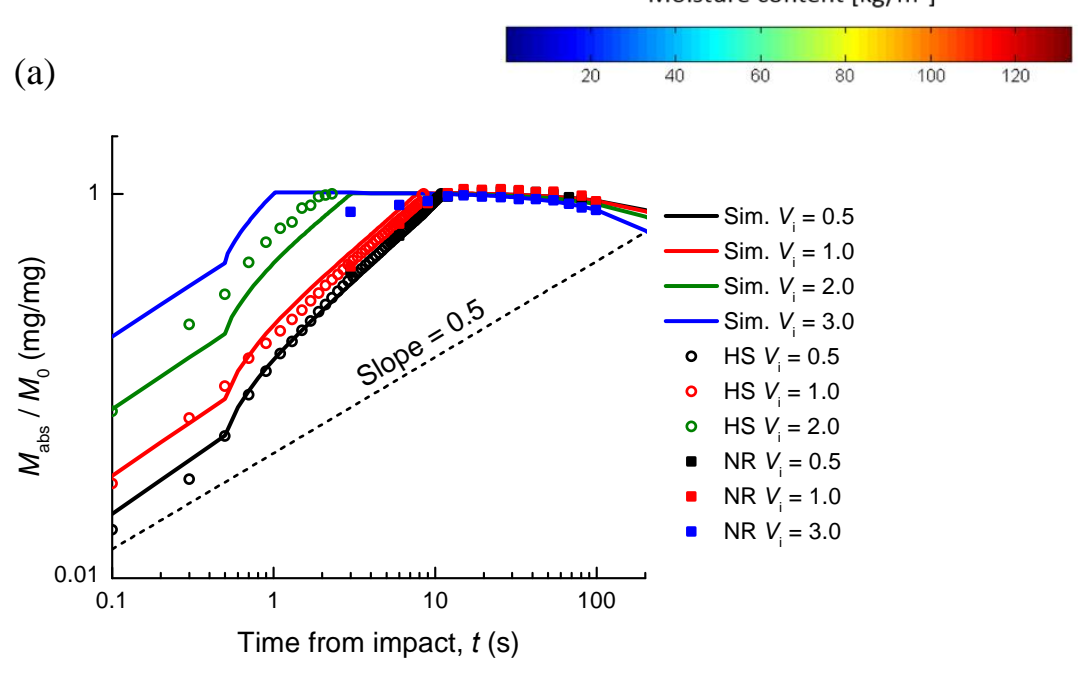

Average moisture content $\left(\mathrm{kg} / \mathrm{m}^{3}\right)$
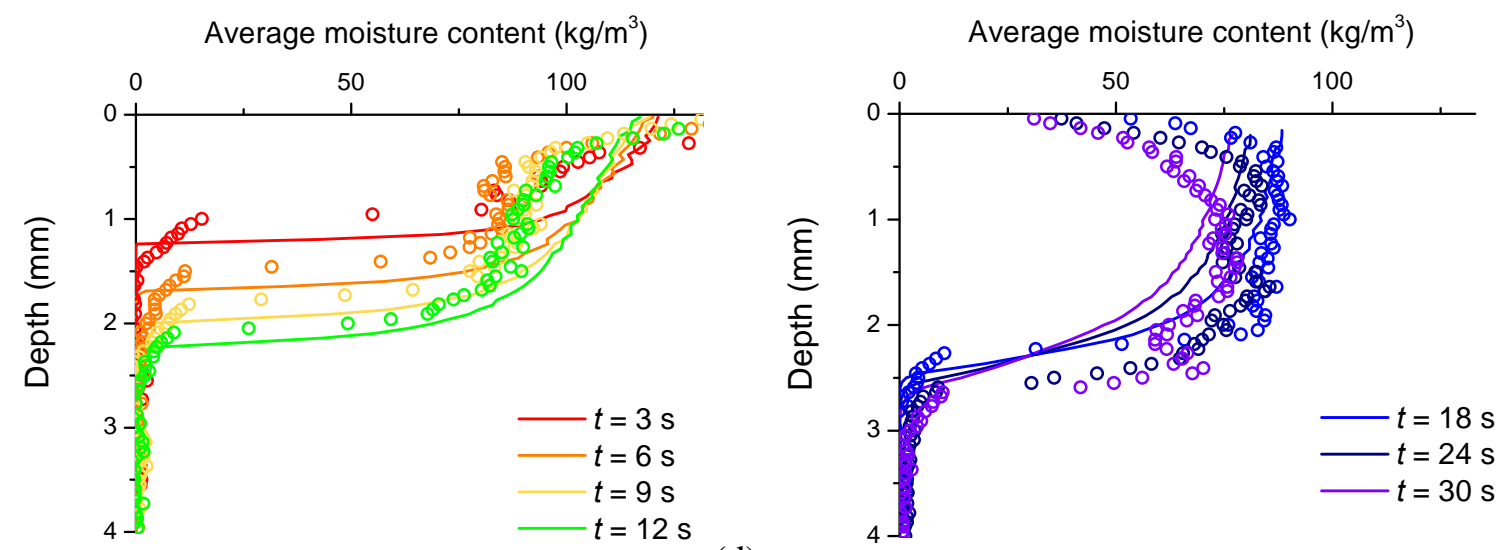

(c)

(d)

Figure 12: (a) Simulated moisture content distribution from droplet uptake, contact area as per impact velocity of $1.0 \mathrm{~m} / \mathrm{s}$, at different times. The dashed line indicates the symmetry line of the modelled geometry. (b) Comparison of measured and simulated absorption mass normalized by initial impinging drop mass versus time in log-log plot for Savonnières for different impact velocities. Average moisture content over sample depth for contact area as per impact velocity of $0.5 \mathrm{~m} / \mathrm{s}$ during (c) absorption and (d) evaporation phase (the circles indicate neutron radiography measurement results, while the lines show simulation data). 
As an example, Figure 12a shows the simulated moisture content distribution during absorption and evaporation for a droplet deposited with an impact velocity of $1 \mathrm{~m} / \mathrm{s}$, yielding for the pinned droplet a constant diameter of $3.5 \mathrm{~mm}$. The images at $t=3$ and $t=6$ seconds show the moisture distribution during the absorption phase before the droplet is depleted. The images at $t=18$ and $t=30 \mathrm{~seconds}$ show the moisture content distributions during the evaporation phase. Figure $12 \mathrm{~b}$ compares the absorbed mass normalized by the initial impinging drop mass versus time in a log-log plot for impact velocity of $V_{\mathrm{i}}=0.5,1,2$ and $3 \mathrm{~m} / \mathrm{s}$, with a constant diameter of the pinned droplet of 2.8, 3.5, 5.3 and $8.2 \mathrm{~mm}$ respectively. For the highest impact velocity, we did not consider splashing, but a uniform spreading equal to the splashing area. All measured data as obtained from high-speed camera and neutron imaging are shown. In first simulations, the absorption process was initiated by imposing a capillary pressure at the droplet contact area equal to the capillary pressure at capillary moisture content $\left(p_{c, d r o p}=10 \mathrm{~Pa}\right)$. This boundary condition however showed a much too fast uptake by the porous substrate. The overprediction of the initial uptake stemmed probably from the assumption that a perfect capillary contact exists between droplet and porous medium when imposing a near-zero capillary pressure, while in reality this contact is not perfect. This contact resistance for fluid transport from droplet to substrate is modelled by imposing a higher capillary pressure ( $p_{c, d r o p}=1 \mathrm{E} 4 \mathrm{~Pa}$ ) at the contact related to a contact moisture content $\left(\mathrm{w} \approx 120 \mathrm{~kg} / \mathrm{m}^{3}\right)$ lower than the capillary moisture content. We impose this contact resistance condition only for the first 0.5 seconds of the absorption process. The reasons for this contact behavior will be discussed later in the discussion section. Figure $12 \mathrm{~b}$ shows that a good agreement between simulation and measurement data is obtained for both the absorption and evaporation phases. The first absorption period with contact resistance is clearly visible and follows nearly a 0.5 slope as predicted by the Washburn relation. This means that, during this period, the uptake process is nearly unidimensional. The uptake process during the absorption phase with perfect capillary contact however does not show this 0.5 slope behavior, indicating a tridimensional uptake process. The simulations also predict correctly the decrease in droplet depletion time with increasing impact velocity.

Figures $12 \mathrm{c}$ and d compare the average moisture content over the sample depth derived from neutron radiography measurements with the numerical results. A satisfactory agreement can be observed for both the absorption and evaporation phases taking the uncertainty on the material properties.

The numerical model allows the prediction of the moisture content profiles over the depth of the porous medium at the center-line during the absorption and evaporation stages at different points of time. At low impact velocity $V_{\mathrm{i}}=0.5 \mathrm{~m} / \mathrm{s}$ (Fig. 13a), the moisture content increases in time with depth during the absorption phase $(t \approx 12 \mathrm{~s}$ ). During the evaporation phase, the moisture content at the surface decreases, while still further penetration of liquid into the porous medium occurs due to redistribution. At high impact velocity $V_{\mathrm{i}}=3 \mathrm{~m} / \mathrm{s}$ (Fig. 13b) the droplet is already depleted at $\mathrm{t} \approx 3 \mathrm{~s}$ 
and evaporation has started. This example shows that droplet spreading as well as droplet depletion and evaporation is highly determined by the impact velocity of the droplet.

(a)

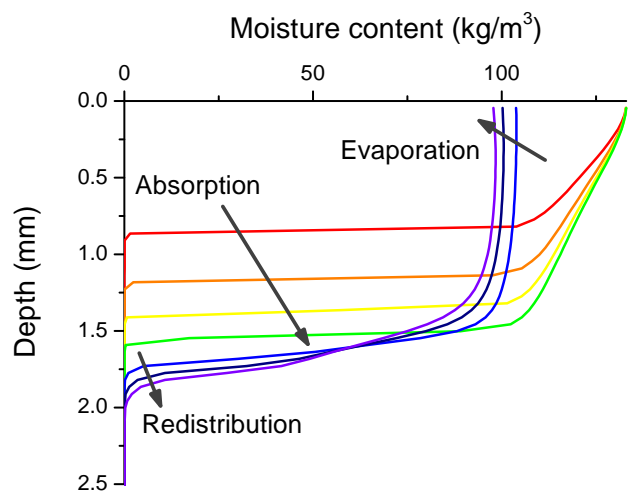

Figure 13: Simulated moisture content at centerline of porous medium over depth for different times at impact velocity of $0.5 \mathrm{~m} / \mathrm{s}$ (a) and $3 \mathrm{~m} / \mathrm{s}$ (b).

\section{Discussion}

Figures 14 and 15 summarize and interpret our observations schematically. The life of a droplet after impact on a porous stone covers different time scales (Figure 14). At short time after drop impact, the droplet spreads showing a dynamic contact angle higher than $90^{\circ}$ indicating a dynamic non-wetting behavior. The droplet then gets pinned showing a dynamic contact angle decreasing sharply after reaching maximum spreading. During this dynamic phase, no liquid mass penetrates into the porous substrate. We explain this behavior by the presence of an air layer between the droplet and the porous substrate, as discussed in [Lee et al. 2015]. During spreading, the presence of this air layer leads to the observed dynamic hydrophobic non-wetting behavior (Fig. 15a). When the contact line velocity decreases reaching maximum spreading, the air layer between droplet and surface can be broken, leading to capillary contact between droplet and porous substrate (Fig 15b). This capillary contact induces high capillary forces on the droplet showing high capillary suction especially by the fine pores. The droplet remains pinned at the contact line at maximum spreading due to these high capillary forces in the fines pores at the droplet edge leading to a change from non-wetting to wetting behavior and a contact angle $<90^{\circ}$. Part of the air remains however entrapped under the pinned droplet. 


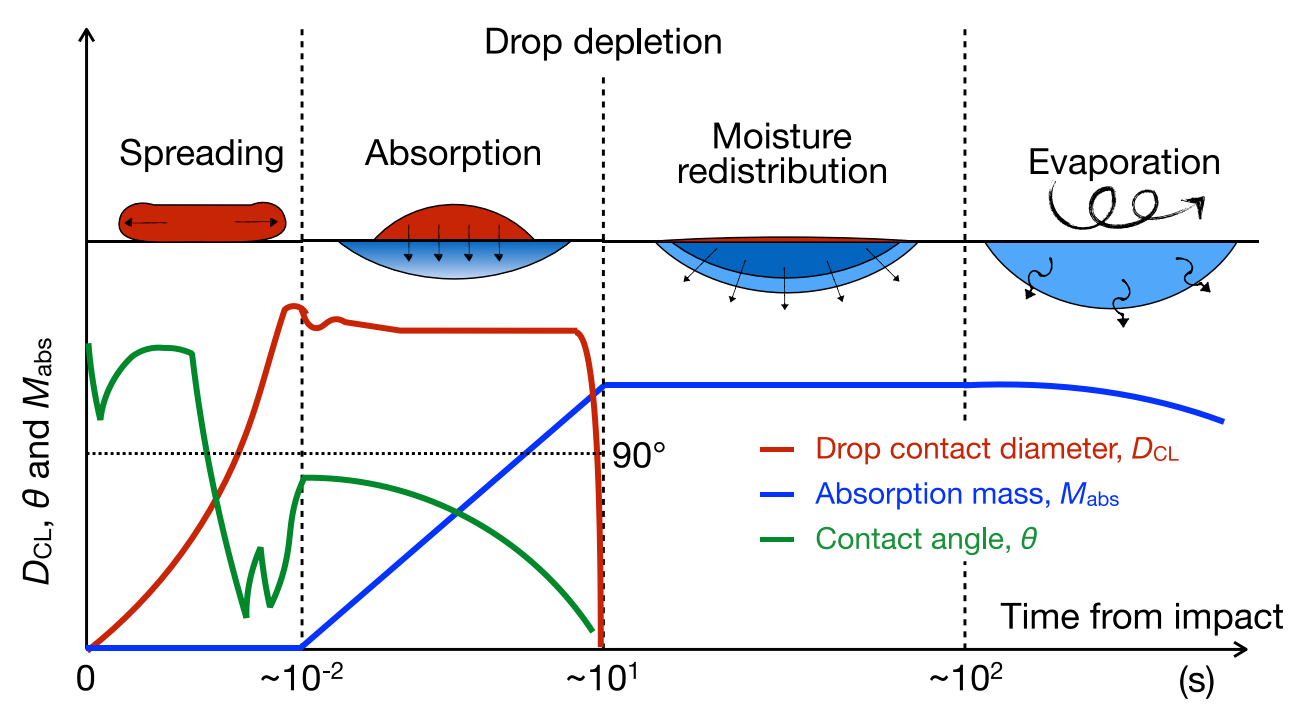

Figure 14. Schematic representation of the life of a droplet after impact on a porous stone over different time scales: spreading, absorption, droplet depletion, moisture redistribution and evaporation. Graphical representation of time evolution of droplet contact line diameter, contact angle and absorbed mass in the porous medium.

During the absorption phase, the droplet remains pinned and the contact angle decreases in a constant contact radius (CCR) mode (Figure 14). With time, the mass absorbed in the stone increases until the droplet is depleted. . The higher contact resistance at the beginning of the adsorption process is attributed to the presence of entrapped air (Figure 15c). With time, this entrapped air will disappear due to air leakage from the contact zone, resulting in a decrease of contact resistance and therefore faster capillary uptake process (Figure 15d). At the end of the absorption phase, the droplet radius decreases due to the complete uptake of the liquid occurring first at the droplet edge. Finally, the droplet is depleted and moisture starts to evaporate from the top surface, while moisture redistribution still goes on deeper into the porous material.

(a) spreading phase

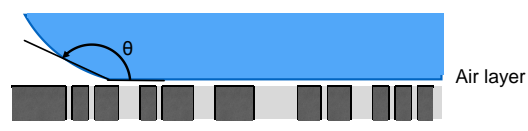

(b) pinning at maximum spreading

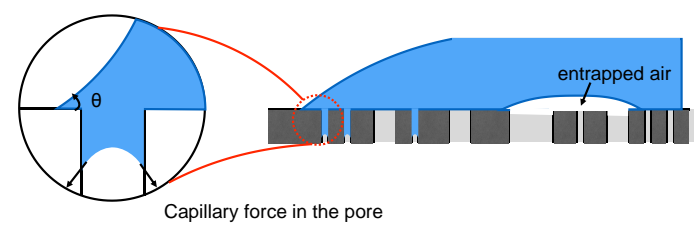

(c) first slow absorption phase

contact resistance due to entrapped air

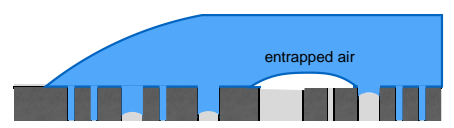

(d) second fast absorption phase capillary uptake

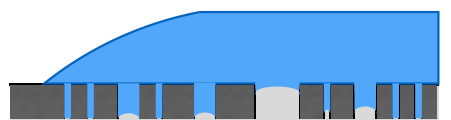

Figure 15. Schematic of dynamic spreading (a), pinning at maximum spreading (b), first (c) and second (d) absorption phase. (a) Presence of an air layer between droplet and porous substrate leads to a non-wetting dynamic spreading characterized by a contact angle $>90^{\circ}$. (b) Droplet gets pinned at 
the contact line at maximum spreading due to capillary forces in fine pores at the droplet edge leading to a wetting behavior and a contact angle $<90^{\circ}$. (c) Slow absorption rate at the beginning of the absorption process due to contact resistance caused by the entrapped air under the pinned droplet. (d) When the entrapped air and contact resistance disappeared, the absorption rate increases due to perfect capillary contact.

\section{Conclusion}

This paper presents an experimental investigation and numerical analysis of the absorption of liquid droplets impacting porous stones. High-speed imaging and neutron radiography are used to quantify moisture absorption in three natural stones of varying porosity and moisture uptake characteristics. Neutron radiography provides quantitative distribution of high resolution moisture content throughout the different phases of the phenomenon. The life of a droplet after impact on a porous stone continues over different time scales. At short time after drop impact the droplet spreads showing a non-wetting dynamic behavior. During this dynamic phase, no liquid mass penetrates into the porous substrate due to the presence of an air layer between the droplet and the porous substrate. At maximum spreading, the air layer between droplet and surface is broken at the contact line leading to capillary contact and pinning of the droplet due to capillary forces in the pores at the droplet edge. As a result, the contact angle changes from a non-wetting to wetting behavior, while air remains entrapped under the pinned droplet. During the absorption phase at larger time scale, the droplet remains pinned and the contact angle decreases in a constant contact radius (CCR) mode. The mass absorbed in the stone increases until the droplet is depleted. The absorption phase is first hindered by the presence of entrapped air, leading to a contact resistance for fluid transport from droplet to substrate. The entrapped air shortly disappears from the contact zone leading to perfect capillary contact between droplet and porous medium. Droplet absorption and depletion happens faster in highly capillary active stones. Evaporation and further redistribution are observed once the droplet is depleted. Droplet spreading as well as droplet depletion and evaporation is highly determined by the impact velocity of the droplet. A finite-element numerical model for isothermal moisture transport in unsaturated porous media is found to capture properly the mass absorption as observed in the experimental data. A good agreement is obtained between the average moisture content over sample depth from neutron radiography measurements and the numerical results, for absorption and evaporation phases.

\section{Acknowledgments}

The authors acknowledge the support of Swiss National Science Foundation grant no. 200021135510. Stefan Carl and Guylaine Desmarais are acknowledged for their support in the preparation and running of the PSI Neutra experiment. 


\section{References}

H. Ardebrant, R.J. Pugh. Wetting studies on silicate minerals and rocks used in bituminous highways, Colloids and Surfaces, 58 (1991) 111-130.

D. Ben Jazia, L. Vonna, G. Schrodj, H. Bonnet, Y. Holl, H. Haidara. Imbibing drops of ethanol/water mixtures in model nanoporous networks with tunable pore structure: deviation from square root to linear time regime imbibition kinetics, Colloids and Surfaces a: Physicochemical and Engineering Aspects, 384 (2011) 643652.

J. Carmeliet, S. Roels. Determination of the isothermal moisture transport properties of porous building materials. Journal of Thermal Envelope and Building Science, 24 (2001) 183-210.

T.H. Chilton, A.P. Colburn. Mass transfer (absorption) coefficients prediction from data on heat transfer and fluid friction, Industrial and Engineering Chemistry, 26 (1934) 1183-1187.

A. Clarke, T.D. Blake, K. Carruthers, A. Woodward. Spreading and imbibition of liquid droplets on porous surfaces, Langmuir, 18 (2002) 2980-2984.

M. Denesuk, G. Smith, B. Zelinski, N. Kreidl, D. Uhlmann. Capillary penetration of liquid droplets into porous materials, Journal of Colloid and Interface Science, 158 (1993) 114-120.

H. Derluyn. Transport and crystallization in porous limestone: neutron - X-ray imaging and poromechanical modeling, [dissertation ETH No. 20673]. Zürich: ETH Zürich, (2012).

H. Derluyn, P. Moonen, J, Carmeliet. Deformation and damage due to drying-induced salt crystallization in porous limestone. Journal of the Mechanics and Physics of Solids 63 (2014) 242-255.

R. Dreesen, M. Dusar. Historical building stones in the province of Limburg (NE Belgium): role of petrography in provenance and durability assessment, Materials Characterization, 53 (2004) 273-287.

T.G. D’Onofrio, H.K. Navaz, B. Markicevic, B.A. Mantooth, K.B. Sumpter. Experimental and numerical study of spread and sorption of VX sessile droplets into medium grain-size sand, Langmuir, 26 (2010) 33173322.

T. Gambaryan-Roisman. Liquids on porous layers: wetting, imbibition and transport processes, Current Opinion in Colloid and Interface Sci., 19 (2014) 320-335.

H. Janssen, B. Blocken, J. Carmeliet. Conservative modelling of the moisture and heat transfer in building components under atmospheric excitation, International Journal of Heat and Mass Transfer, 50 (2007) 1128-1140.

K.P. Hapgood, J.D. Litster, S.R. Biggs, T. Howes. Drop penetration into porous powder beds, Journal of Colloid and Interface Science, 253 (2002) 353-366.

S.Y. Jung, S. Lim, S.J. Lee. Investigation of water seepage through porous media using X-ray imaging technique, Journal of Hydrology, 452-453 (2012) 83-89.

J.B. Lee, N. Laan, K.G. de Bruin, G. Skantzaris, D. Derome, J. Carmeliet, D. Bonn. Universal rescaling of droplet impact on smooth and rough surfaces, J. Fluid Mech. 786 (2016), 10.1017/jfm.2015.620.

J.B. Lee, D. Derome, J. Carmeliet. Drop impact on natural porous stones, under revision (after review) by J. Colloid and Interface Science (2015).

E.H. Lehmann. Recent improvements in the methodology of neutron imagin, Pramana, 71 (2008) 653-661.

M. Marengo, C. Antonini, I.V. Roisman, C. Tropea. Drop collisions with simple and complex surfaces, Current Opinion in Colloid \& Interface Science, 16 (2011) 292-302. 
B. Markicevic, H.K. Navaz, Primary and Secondary Infiltration of Wetting Liquid Sessile Droplet into Porous Medium, Transport in Porous Media, 85 (2010) 953-974.

B. Markicevic, H. Li, Y. Sikorski, A.R. Zand, M. Sanders, H.K. Navaz. Infiltration time and imprint shape of a sessile droplet imbibing porous medium, Journal of Colloid and Interface Science, 336 (2009) 698-706.

A. Marmur. Drop penetration into a thin porous medium, Journal of Colloid and Interface Science, 123 (1988) $161-169$.

P. Moonen, Contiuous-Discontinuous Modelling of Hygrothermal Damage Processes in Porous Media., TU Delft, Delft University of Technology, 2009.

H.K. Navaz, B. Markicevic, A.R. Zand, Y. Sikorski, E. Chan, M. Sanders, T.G. D’Onofrio. Sessile droplet spread into porous substrates - determination of capillary pressure using a continuum approach, Journal of Colloid and Interface Science, 325 (2008) 440-446.

T. Okayama, D.S. Keller, P. Luner. The wetting of calcite surfaces, The Journal of Adhesion, 63 (1997) 231252.

N.C. Reis, R.F. Griffiths, M.D. Mantle, L.F. Gladden. Investigation of the evaporation of embedded liquid droplets from porous surfaces using magnetic resonance imaging, International Journal of Heat and Mass Transfer, 46 (2003) 1279-1292.

N.C. Reis Jr., R.F. Griffiths, J.M. Santos. Numerical simulation of the impact of liquid droplets on porous surfaces, Journal of Computational Physics, 198 (2004) 747-770.

N.C. Reis Jr., R.F. Griffiths, M.D. Mantle, L.F. Gladden, J.M. Santos. MRI investigation of the evaporation of embedded liquid droplets from porous surfaces under different drying regimes, International Journal of Heat and Mass Transfer, 49 (2006) 951-961.

M. Rodriguez-Valverde, M. Cabrerizo-Vilchez, P. Rosales-Lopez, A. Paez-Duenas, R. Hidalgo-Alvarez. Contact angle measurements on two (wood and stone) non-ideal surfaces, Colloids and Surfaces a: Physicochemical and Engineering Aspects, 206 (2002) 485-495.

S. Roels, J. Carmeliet, H. Hens. Modelling unsaturated moisture transport in heterogeneous limestone (Part 1. Mesoscopic approach), Transp. Porous Med. 52 (2003) 333-350.

M. Sedighi Gilani, M. Griffa, D. Mannes, E, Lehmann, J. Carmeliet, D. Derome. Visualization and quantification of liquid water transport in softwood by means of neutron radiography, International Journal of Heat and Mass Transfer, 55 (2012) 6211-6221.

M.P. Willis, B.A. Mantooth, T.A. Lalain. Novel Methodology for the Estimation of Chemical Warfare Agent Mass Transport Dynamics. Part II: Absorption, J. Phys. Chem. C 116 (2011) 546-554

A.L. Yarin. Drop impact dynamics: splashing, spreading, receding, bouncing..., Annual Review of Fluid Mechanics, 38 (2006) 159-192. 


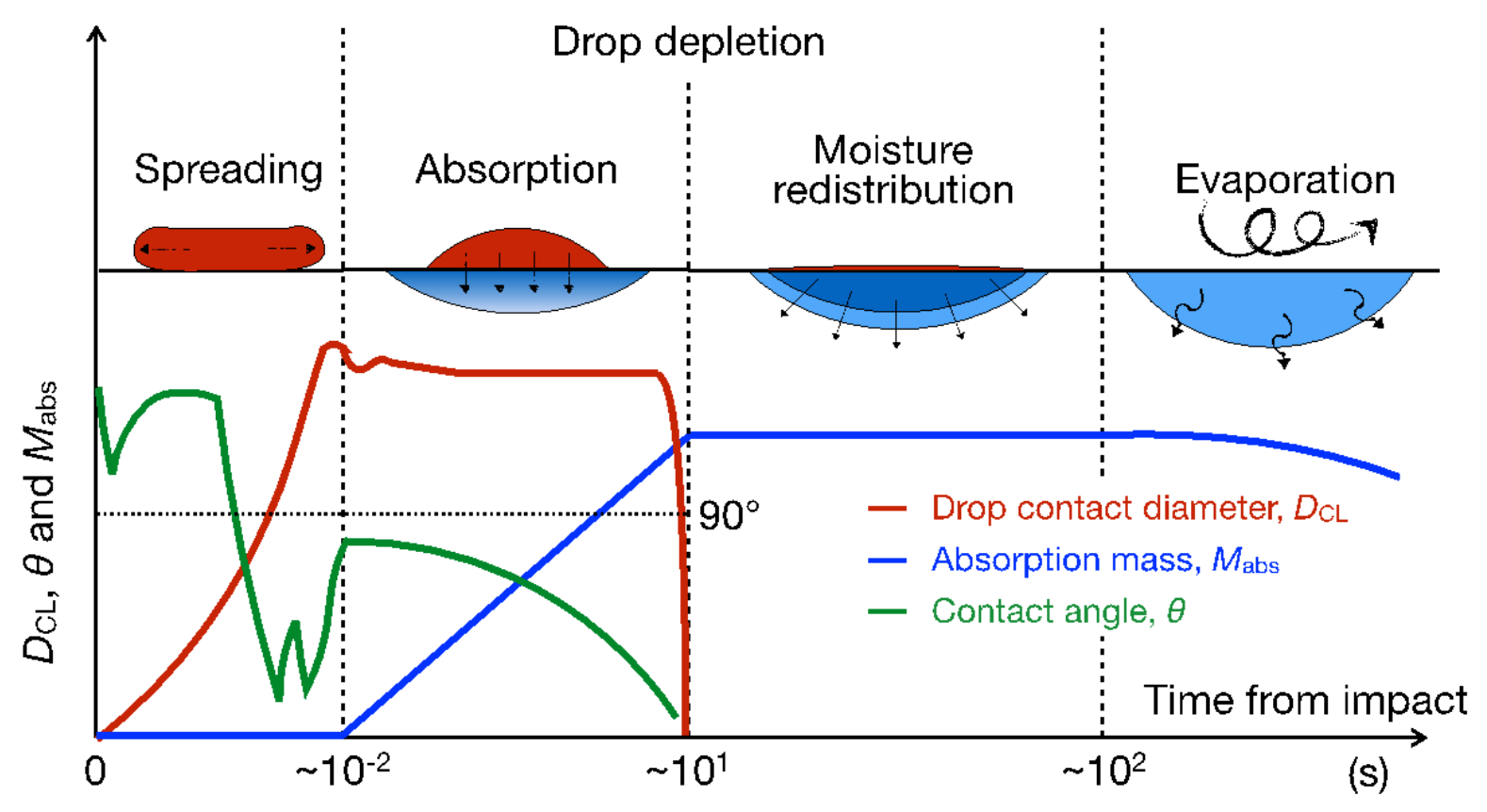

